\title{
Factors affecting determination of the optimal ketamine dose for pediatric sedation
}

\section{Ji Young Min, Jeong-Rim Lee, Hyun IL Kim, Hyo Jin Byon}

Department of Anesthesiology and Pain Medicine, Anesthesia and Pain Research Institute, Yonsei University College of Medicine, Seoul, Korea

Objective Children are sedated before undergoing diagnostic imaging tests in emergency medicine or pediatric sedation anesthesia units. The aim of this study was to identify variables potentially affecting the dose of ketamine required for induction of sedation in pediatric patients undergoing diagnostic imaging.

Methods This retrospective study included children aged 0 to 18 years who underwent sedation with ketamine for computed tomography or magnetic resonance imaging in the pediatric sedation anesthesia unit of a tertiary medical center between January 2011 and August 2016. The children's hemodynamic status and depth of sedation were monitored during the examination. We recorded data on demographics, categories of imaging tests, ketamine doses administered, adverse events, respiratory interventions, and duration of sedation. Data for patients who experienced adverse events were excluded.

Results Sixty-six patients were included in the final analysis. Univariate linear regression analysis revealed that patient age, height, and body surface area (BSA) affected the sedative dose of ketamine administered. These three variables showed multicollinearity in multivariate linear regression analysis and were analyzed in three separate models. The model with the highest adjusted $\mathrm{R}$-squared value suggested the following equation for determination of the dose of ketamine required to induce sedation: ketamine dose $(\mathrm{mg})=-1.62+0.7 \times$ age $($ months $)+36.36 \times \mathrm{BSA}\left(\mathrm{m}^{2}\right)$.

Conclusion Variables such as age and BSA should be considered when estimating the dose of ketamine required for induction of sedation in pediatric patients.

Keywords Ketamine; Anesthesia; Patient care

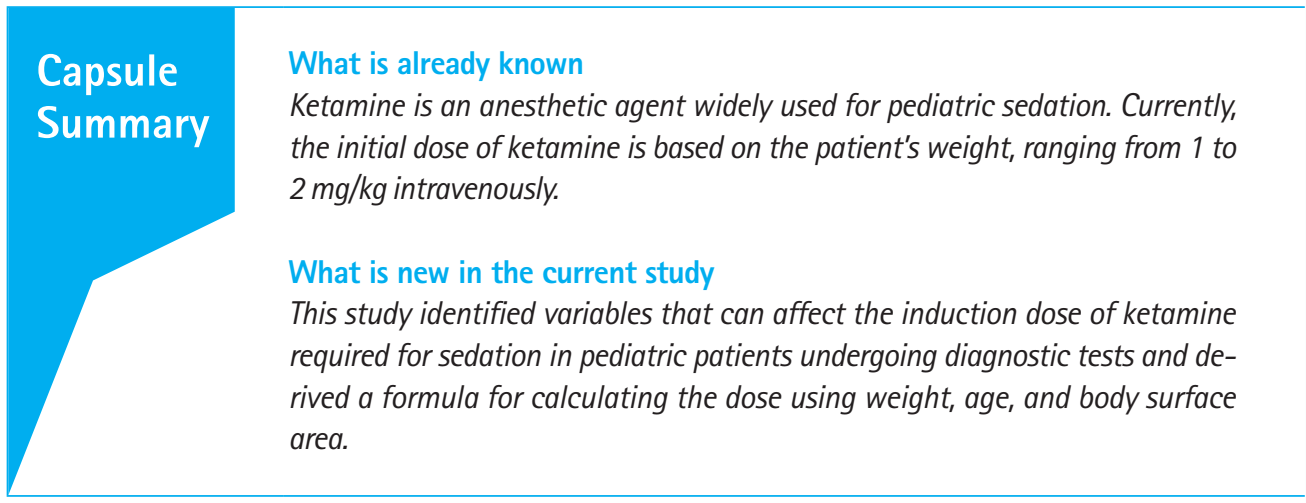

elSSN: 2383-4625

Received: 27 February 2018

Revised: 25 April 2018

Accepted: 5 May 2018

Correspondence to: Hyo Jin Byon Department of Anesthesiology and Pain Medicine, Anesthesia and Pain Research Institute, Severance Hospital, Yonsei University College of Medicine, 50-1 Yonsei-ro, Seodaemun-gu, Seoul 03722, Korea

E-mail: JINOBEN@yuhs.ac ORCID

http://orcid.org/0000-0002-5400-6155

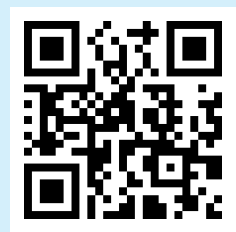

How to cite this article:

Min JY, Lee JR, Kim HI, Byon HJ. Factors affecting determination of the optimal ketamine dose for pediatric sedation. Clin Exp Emerg Med 2019;6(2):119-124.

This is an Open Access article distributed under the terms of the Creative Commons Attribution Non-Commercial License (http:// creativecommons.org/licenses/by-nc/4.0/). 


\section{INTRODUCTION}

Examinations using diagnostic imaging modalities such as computed tomography and magnetic resonance imaging are becoming increasingly common in pediatric sedation and anesthesia units. Therefore, it is becoming important to ensure adequate sedation of patients undergoing such examinations.'

If the level of sedation is inadequate, a child may move during the imaging procedure, which interferes with the examination, whereas deep sedation may lead to complications, such as apnea and hemodynamic instability. Therefore, appropriate doses of anesthetic agents are essential for successful examination of children undergoing diagnostic imaging tests. ${ }^{2,3}$

Ketamine is an anesthetic agent that is widely used for pediatric sedation. Currently, the initial dose of ketamine is based on the patient's weight and is in the range of $1.0-2.0 \mathrm{mg} / \mathrm{kg}$ intravenously, and a further dose is considered if the duration of examination is extended or if the patient is noticed to be about to wake up or shows movement. However, the optimal dose of ketamine can vary for a number of reasons, including the patient's medical condition, the environment in the examination room, and the type of diagnostic test being performed. Furthermore, anthropometric variables other than weight, such as age, height, and body surface area (BSA), could influence the optimal dose of ketamine. Therefore, a specific guideline for determining the optimal dose of ketamine could help to improve the success rate of diagnostic examinations and decreasing the risk of complications. ${ }^{4-6}$

The aim of this study was to identify variables that could affect the dose of ketamine required for induction of sedation in pediatric patients undergoing diagnostic tests and to devise a formula for calculating the optimal dose using those variables.

\section{METHODS}

This study was approved by the Institutional Ethical Committee (IRB number: 4-2016-0733). Informed consent was waived due to the retrospective nature of the study. This retrospective observational study included children aged 0 to 18 years who were sedated in the pediatric sedation and anesthesia unit at our tertiary medical center from January 2011 to August 2016. The medical records of patients who received ketamine for sedation during an imaging procedure were evaluated.

Pediatric inpatients and outpatients who were referred for diagnostic imaging (computed tomography or magnetic resonance imaging) under sedation were included in the study. An anesthesiologist and a nurse monitored each patient's hemodynamic status and depth of sedation during the examination. Monitoring devices for pulse oximetry, noninvasive measurement of blood pressure, electrocardiography, and capnography were connected to the child before the examination. Glycopyrrolate $0.004 \mathrm{mg} / \mathrm{kg}$ was injected intravenously and ketamine $1 \mathrm{mg} / \mathrm{kg}$ was administered for intravenous sedation. Loss of consciousness was judged by disappearance of the eyelash reflex and no resistance to fitting of the mask. If the patient remained conscious, an additional $1-\mathrm{mg} / \mathrm{kg}$ dose of ketamine was administered. The sedation level was maintained at 5 to 6 points on the Modified Ramsay Sedation Scale. When the imaging study was finished, the child was taken to the recovery room. The child was discharged from the recovery room if hemodynamic values were maintained within $\pm 20 \%$ of the pre-sedation values, the modified Aldrete post anesthesia recovery score remained over 9 points, and if the child was fully awake.

We recorded the patient characteristics, including weight, age, height, and BSA. The type of imaging test, doses of sedatives administered until unconsciousness, adverse events, respiratory interventions for sedation, and duration of sedation were also recorded. Data for patients who experienced an adverse event, such as desaturation or hemodynamic instability, were excluded from the analysis. BSA was defined as follows: $0.016667 \times 0.5 \times$ body weight $(\mathrm{kg}) \times 0.5 \times$ height $(\mathrm{cm})$.

\section{Study outcomes and data analyses}

The primary aim of the study was to identify the factors most closely related to the dose of ketamine required for induction of sedation. The relationships of sex, age, weight, height, and BSA with the dose of ketamine required for induction of sedation were analyzed using linear regression. The regression equation was then used in multivariate analysis. The variance inflation factor was checked for multiple collinearity before performing the multivariate linear regression. Multicollinearity was determined to be present when the variance inflation factor was $>10$. If multiple collinearity exists between independent variables, they cannot be analyzed in a single model; therefore, the study variables were analyzed using three different models. Each model was inspected visually for linearity, heteroscedasticity, and normality of the residuals. All analyses were performed using SAS ver. 9.4 (SAS Institute Inc., Cary, NC, USA).

\section{RESULTS}

Two hundred and ninety-six of 1,200 pediatric patients who received ketamine for sedation during diagnostic imaging had complete medical records available for analysis. After exclusion of the records for 230 patients who either did not have accurate 
information available for height and weight or experienced adverse events, data for 66 patients were available for inclusion in the analysis. The demographic characteristics of the patients are shown in Table 1. The average dose of ketamine required for in-

Table 1. Patient demographics and clinical data

\begin{tabular}{lc}
\hline Characteristics & Value \\
\hline Sex (male/female) & $41 / 25$ \\
Median age (mo) & $1.45(0.4-3.3)$ \\
Height $(\mathrm{cm})$ & $54.6 \pm 10.7$ \\
Body weight $(\mathrm{kg})$ & $4.8 \pm 2.6$ \\
Type of imaging procedure & \\
CT & $24(36.3)$ \\
MRI & $42(63.7)$ \\
CT+MRI & 0 \\
Sedation time (min) & $21.7 \pm 12.1$ \\
\hline
\end{tabular}

Value are presented as number, median (interquartile range), mean \pm standard deviation, or number (\%).

$\mathrm{CT}$, computed tomography; $\mathrm{MRI}$, magnetic resonance imaging.
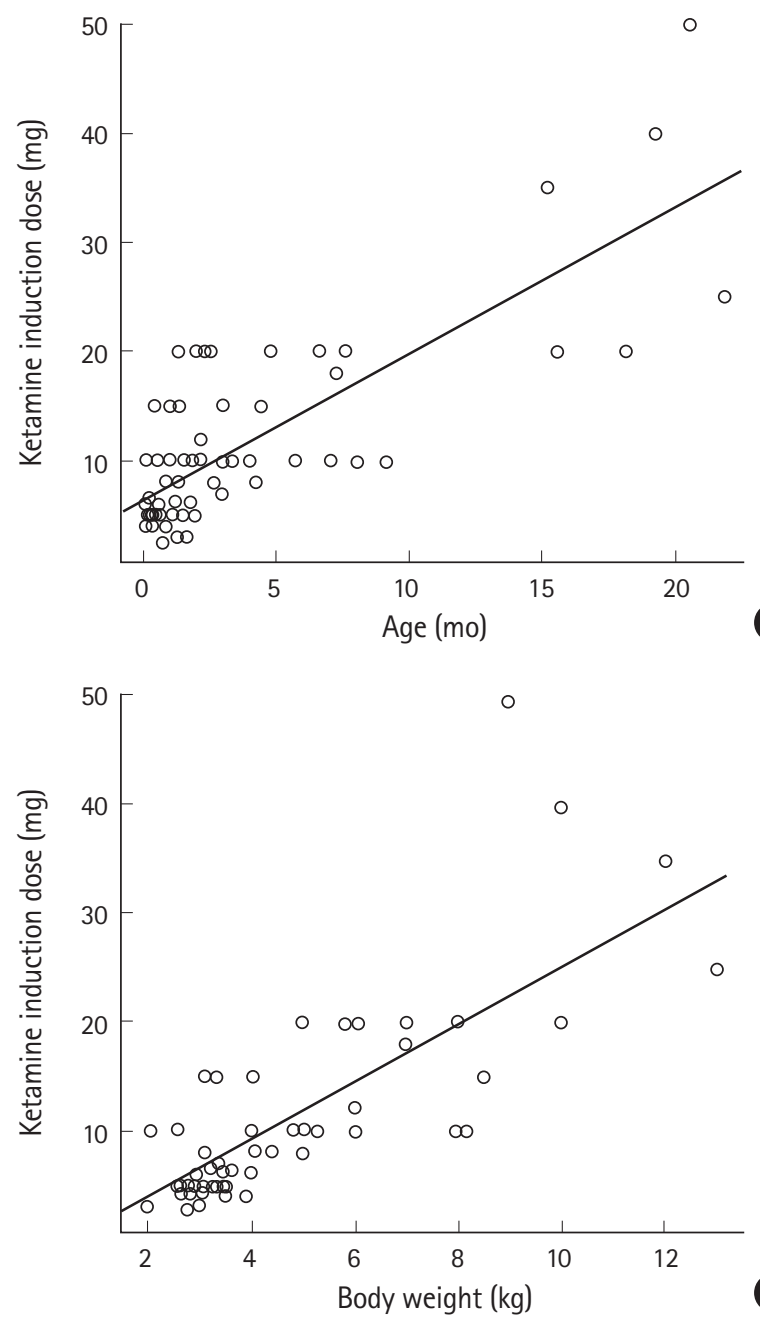

A

Fig. 1. Scatter plot about linear correlation. (A-D) Correlation between various characteristic and induction dose of ketamine for sedation. This shows a tendency toward increasing slope line with increasing (A) age, $(B)$ height, $(C)$ body weight, and (D) body surface area (BSA). duction of sedation was $2.29 \mathrm{mg} / \mathrm{kg}$. In the univariate linear regression analyses, age, height, body weight, and BSA were identified to be factors affecting the dose required (Table 2 and Fig. 1).

The variance inflation factor was checked for multiple collinearity before performing the multivariate linear regression. The independent variables of height, weight, and BSA showed multiple collinearity, so it was not possible to analyze them in the

Table 2. Univariate regression analysis to identify independent factors affecting induction dose of ketamine for sedation $(n=66)$

\begin{tabular}{lcc}
\hline Variable & $\beta(\mathrm{SE})$ & P-value \\
\hline Sex & $-2.000(2.257)$ & 0.379 \\
Age $(\mathrm{mo})$ & $1.336(0.128)$ & $<0.001$ \\
Height $(\mathrm{cm})$ & $0.646(0.066)$ & $<0.001$ \\
Body weight $(\mathrm{kg})$ & $2.657(0.272)$ & $<0.001$ \\
BSA $\left(\mathrm{m}^{2}\right)$ & $71.409(6.991)$ & $<0.001$ \\
\hline
\end{tabular}

$\mathrm{SE}$, standard error; BSA, body surface area.
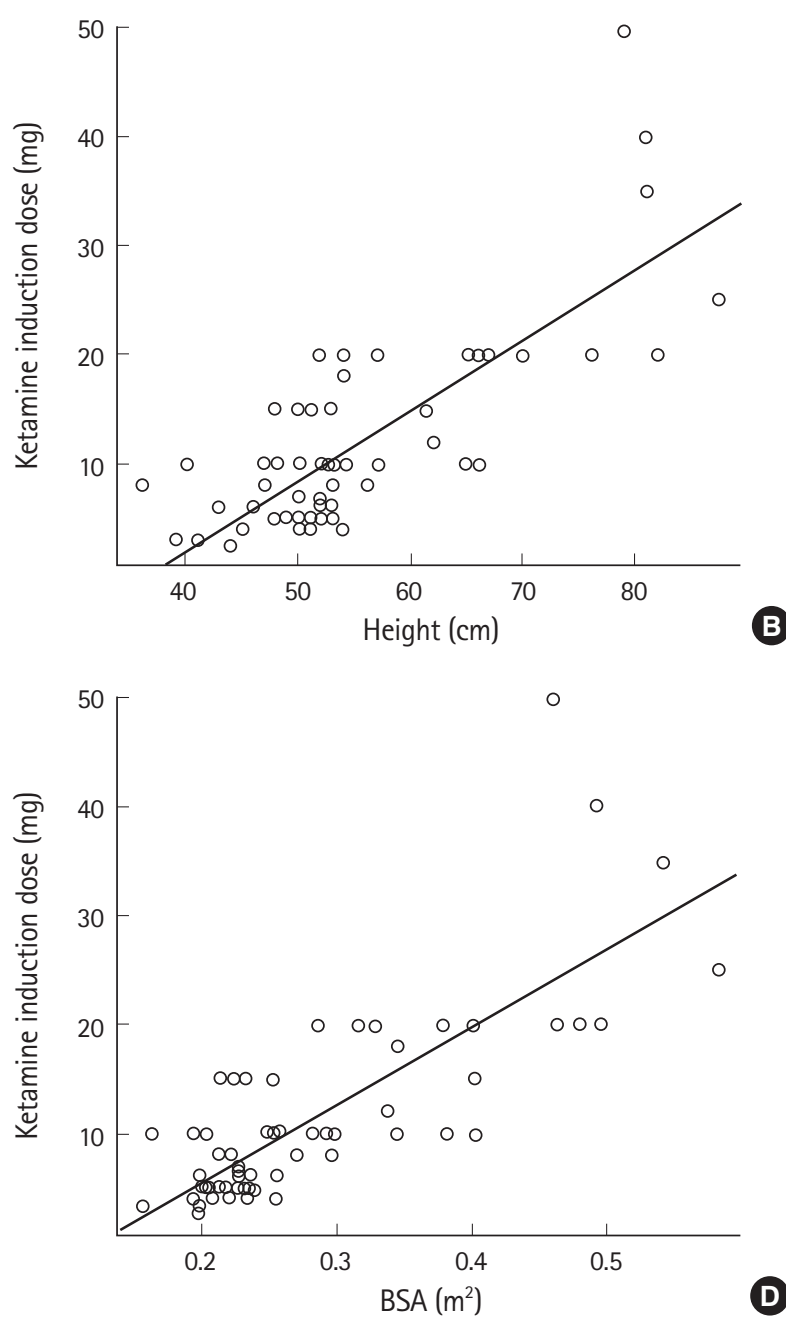

D 
Table 3. Calculation of variance inflation factor to check for multicollinearity

\begin{tabular}{lc}
\hline Variable & Variance inflation factor \\
\hline Age & 4.581 \\
Height & 50.954 \\
Body weight & 442.738 \\
Body surface area $\left(\mathrm{m}^{2}\right)$ & 713.506 \\
\hline
\end{tabular}

Multi-collinearity exists if the variance inflation factor is $>10$.

Table 4. Multivariate linear regression analysis of age and other variables including height, body weight, and BSA

\begin{tabular}{lcc}
\hline & $\beta$ (standard error) & P-value \\
\hline Model 1 & Adjusted R-squared value $=0.651$ \\
Intercept & $-7.982(5.912)$ & 0.182 \\
Age (mo) & $0.812(0.246)$ & 0.002 \\
Height (cm) & $0.299(0.121)$ & 0.016 \\
Model 2 & Adjusted R-squared value $=0.651$ \\
Intercept & $2.494(1.796)$ & 0.170 \\
Age (mo) & $0.825(0.241)$ & 0.001 \\
Body weight $(\mathrm{kg})$ & $1.213(0.492)$ & 0.016 \\
Model 3 & Adjusted R-squared value $=0.658$ \\
Intercept & $-1.620(3.062)$ & 0.599 \\
Age $(\mathrm{mo})$ & $0.749(0.247)$ & 0.004 \\
BSA (m²) & $36.359(13.294)$ & 0.008
\end{tabular}

BSA, body surface area.

same model at the same time (Table 3). Therefore, the relationship between patient age and each variable was analyzed using three different models (Table 4). Model 3, in which age and BSA were analyzed together, had the highest adjusted R-squared value at 0.658 ; therefore, we chose model 3 , which suggested the following equation for determining the dose of ketamine required for induction of sedation taking into account age and BSA:

Dose of ketamine to induce sedation $(\mathrm{mg})=-1.62+0.7 \times$ age (mo)+36.36 $\times$ BSA $\left(\mathrm{m}^{2}\right)$

Additionally, to verify a normal distribution and iso-dispersion of residuals, a residual analysis was performed using studentized residuals. As shown in Fig. 2, the dots appear to be randomly distributed with no particular tendency centering around zero. The trend is centered around zero but the variance around zero is also scattered uniformly and randomly. To evaluate the skewness of the distribution, we constructed a probability-probability plot (Fig. 3). The points on this plot form a nearly linear pattern, indicating that the normal distribution is a good model for this dataset.

\section{DISCUSSION}

In this study, we reviewed the medical records of children who were successfully sedated with ketamine and performed a regres-

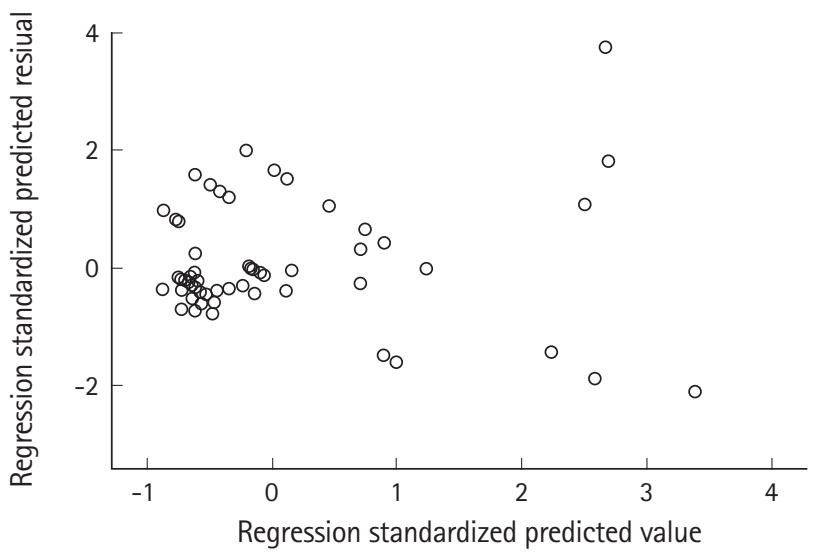

Fig. 2. Scatter plot. Residual plot. Dependent variable: induction dose of ketamine for pediatric sedation. The dots appear randomly distributed with no particular tendency centering around zero.

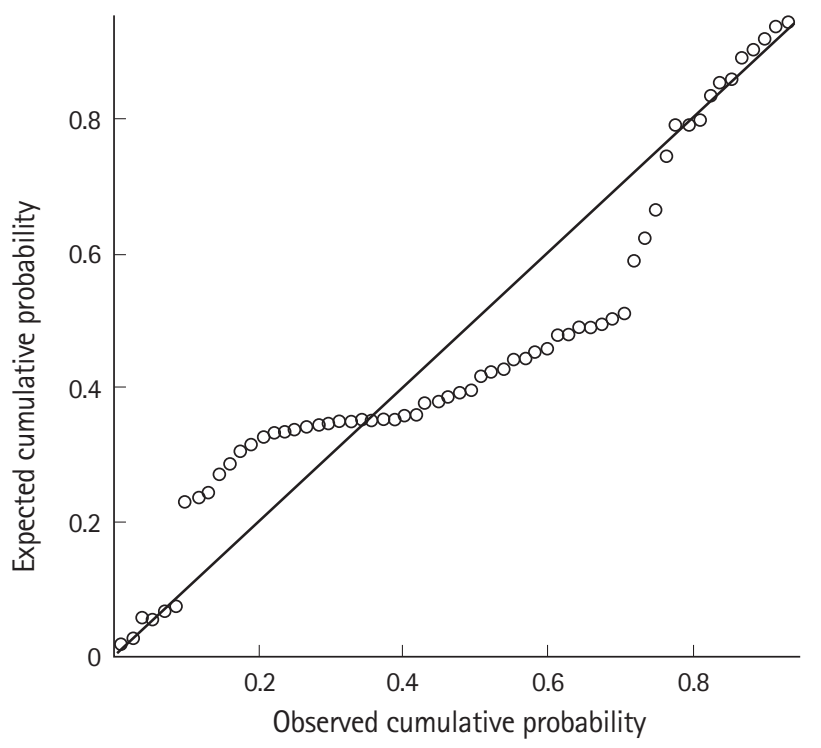

Fig. 3. P-P plot of regression standardized residual. Probability plot. Dependent variable: induction dose of ketamine for pediatric sedation. The points on this plot form a nearly linear pattern, which indicates that the normal distribution is a good model for this dataset.

sion analysis of the relationship between the dose of ketamine and several anthropometric characteristics. The regression analysis showed that the dose of ketamine required for induction of sedation increased with patient age, height, weight, and BSA. The multiple regression analysis indicated that the appropriate dose of ketamine for induction of sedation in children can be calculated accurately using the following formula:

$-1.62+0.7 \times$ age $(\mathrm{mo})+36.36 \times \mathrm{BSA}\left(\mathrm{m}^{2}\right)$.

The optimal dose of ketamine required for sedation is also associated with other anthropometric characteristics, including age, height, and BSA. According to the current guideline, the required 
dose of ketamine is determined on the basis of weight; an intravenous loading dose of $1-2 \mathrm{mg} / \mathrm{kg}$ is used in children. ${ }^{7.8}$ Linear regression analysis in this study identified age, BSA, and height to be significant factors for determining the dose. The most explicable regression equation was used to devise a formula that could determine the dosage of ketamine required for pediatric sedation. If the dosage is determined considering only one factor, the dose range may be too wide. Furthermore, children may be susceptible to small dose changes, which could make it difficult to identify the appropriate dose. Physicians who are inexperienced in pediatric sedation may find it particularly difficult to select the appropriate dose. Consideration of other patient factors, including weight, can increase the accuracy and success rate of sedation.

In this study, we found that it was useful to include BSA in the formula used to estimate the dosage of ketamine required for pediatric sedation. In children, many physical factors, including weight, can affect the pharmacokinetics and pharmacodynamics of an anesthetic agent. Therefore, these factors need to be considered in combination, rather than considering weight in isolation. ${ }^{9-12}$ An earlier study that calculated the dose of propofol required for sedation also considered weight in addition to age. ${ }^{13}$ However, for ketamine, only weight has been considered until now in research concerning the optimal dose required for sedation.

We did not include patient age as a variable in the formula. Body composition, function, and degree of protein binding vary according to age, so the response to administration of the same amount of medication is age-dependent. Therefore, the optimal dose should also vary according to age. Calculating the dose considering age and weight together rather than weight alone will help to establish a guideline that takes the clinical response into account. However, it is not easy to reflect age in an objective manner. The existing guideline suggests that children need a greater dose per kilogram than adults, and there is no formula for calculating the dose by substituting a specific age or other factor(s). Unlike in the existing guideline, the range of selectable doses can be reduced by reflecting age as a numeric item, as in the equation presented here.

Ketamine is a derivative of cyclohexamine that produces sedation and analgesia by separating the cerebral cortex from the limbic system of the brain while preserving the body's reflexes, including the vomiting and laryngeal reflexes. In the existing guideline, the suggested induction dose is determined on the basis of weight alone. However, there is wide patient-to-patient variability in the response to ketamine. Therefore, it is not appropriate to consider only weight when determining the optimal induction dose for sedation. This is the first study to show that the optimal ketamine dose can be calculated using other factors, in- cluding body weight. However, even if the guideline presented in this study is applied, the possibility of an adverse event due to ketamine cannot be ruled out, so precautionary measures are still necessary during sedation using ketamine.

This study has some limitations. First, the optimal dosage of a drug should be determined with a focus on preventing side effects by minimizing overdose; the dose of ketamine used in this study $(1 \mathrm{mg} / \mathrm{kg}$ ) may be appropriate for some patients, but may be an overdose in some patients despite achieving the desired sedation level. Second, the proposed formula could be cumbersome to use when administering ketamine in routine practice because it requires additional calculation of BSA. Third, the patients had various underlying medical conditions. Fourth, the study relied on accurate documentation of medical records, which may have been inadequate. However, this shortcoming may be offset by the use of objective measurements of sedation depth, such as loss of the eyelash reflex and acceptance of the face mask, during the study period. Fifth, various anesthesiologists performed the measurements. Judgment of patient movement and level of sedation may vary between operators, and the extent to which this variation may have influenced the findings of the study is unknown.

In summary, the anthropometric variables of age, height, and BSA should be taken into account when estimating the dose of ketamine required for induction of anesthesia in pediatric patients. The equation proposed here may be helpful for determining the dosages required for pediatric sedation. However, further clinical investigations are required to validate its effectiveness.

\section{CONFLICT OF INTEREST}

No potential conflict of interest relevant to this article was reported.

\section{ACKNOWLEDGMENTS}

This research was supported by a grant of the Korea Health Technology R\&D Project through the Korea Health Industry Development Institute (KHIDI), funded by the Ministry of Health \& Welfare, Republic of Korea (HI16C0793).

\section{REFERENCES}

1. Arlachov Y, Ganatra RH. Sedation/anaesthesia in paediatric radiology. Br J Radiol 2012;85:e1018-31.

2. Ramaiah $\mathrm{R}$, Bhananker $\mathrm{S}$. Pediatric procedural sedation and analgesia outside the operating room: anticipating, avoiding and managing complications. Expert Rev Neurother 2011;11: 
755-63.

3. Slovis TL. Sedation and anesthesia issues in pediatric imaging. Pediatr Radiol 2011;41 Suppl 2:514-6.

4. Dallimore D, Herd DW, Short T, Anderson BJ. Dosing ketamine for pediatric procedural sedation in the emergency department. Pediatr Emerg Care 2008;24:529-33.

5. Morton NS. Ketamine for procedural sedation and analgesia in pediatric emergency medicine: a UK perspective. Paediatr Anaesth 2008;18:25-9.

6. Green SM, Hummel CB, Wittlake WA, Rothrock SG, Hopkins GA, Garrett W. What is the optimal dose of intramuscular ketamine for pediatric sedation? Acad Emerg Med 1999;6:21-6.

7. Heilbrunn BR, Chang TP, Liu DR. A retrospective comparison of ketamine dosing regimens for pediatric procedural sedation. Eur J Emerg Med 2015;22:111-6.

8. Kannikeswaran N, Lieh-Lai M, Malian M, Wang B, Farooqi A, Roback MG. Optimal dosing of intravenous ketamine for pro- cedural sedation in children in the ED-a randomized controlled trial. Am J Emerg Med 2016;34:1347-53.

9. Sawyer M, Ratain MJ. Body surface area as a determinant of pharmacokinetics and drug dosing. Invest New Drugs 2001; 19:171-7.

10. Johnson TN. The problems in scaling adult drug doses to children. Arch Dis Child 2008;93:207-11.

11. Sharkey I, Boddy AV, Wallace $H$, et al. Body surface area estimation in children using weight alone: application in paediatric oncology. Br J Cancer 2001;85:23-8.

12. Lack JA, Stuart-Taylor ME. Calculation of drug dosage and body surface area of children. Br J Anaesth 1997;78:601-5.

13. Jasiak KD, Phan $H$, Christich AC, Edwards CJ, Skrepnek GH, Patanwala AE. Induction dose of propofol for pediatric patients undergoing procedural sedation in the emergency department. Pediatr Emerg Care 2012;28:440-2. 